BULLETIN OF THE

AMERICAN MATHEMATICAL SOCIETY

Volume 77, Number 2, March 1971

\title{
ON A THEOREM OF B. VON KEREKJÁRTÓ
}

\author{
BY PING-FUN LAM
}

Communicated by O. G. Harrold, July 20, 1970

This is the summary of a more detailed study on transformation groups on a metric phase space $X$, which is equicontinuous except for a set $N(X)$ which is topologically sufficiently small compared to its complement. Kerékjártó [7] (1934) gave the following results. Let $f$ be an orientation preserving self-homeomorphism of the 2-sphere $S^{2}$ whose powers (both positive and negative) are equicontinuous except for a finite number of points. Then

(A) The number of exceptional points is at most 2.

(B) For the no exceptional point case, $f$ is topologically conjugated to a rotation about a diameter; for the one exceptional point case, $f$ is topologically conjugated to the homeomorphism obtained from extending the translation on the plane by adding the fixed point $\{\infty\}$; for the two exceptional points case, $f$ is topologically conjugated to the homeomorphism obtained from extending the dilatation on the plane by adding the fixed point $\{\infty\}$.

In this paper we, among other things, obtain an extension of previous works on generalization of (A) by Homma-Kinoshita [5], Kaul [6], and Gray-Roberson [4]. For the difficulty of extending (B) to more general cases consult Montgomery-Zippen [10, p. 229] and Kinoshita [8].

We assume in this announcement that $(X, T, \pi)$ is a transformation group [3] and $X$ is a locally compact metric space with metric $d$.

THEOREM 1. The set of nonequicontinuous points $N(X)=N(X, d, T)$ is always a countable union of closed invariant sets in $X(x \in X$ is said to be equicontinuous if for every $\epsilon>0$, there exists $\delta>0$ such that $d(x, y)$ $<\delta$ implies $d(x t, y t)<\epsilon$ for all $t \in T$, and the set of all such points is denoted by $E(X)=E(X, d, T))$.

Definition. A subset $Y \subset X$ is said to be indivisible by $T$, if, for every sequence $\left\{t_{n}\right\}$ in $T$ such that there is $z \in Y, \lim _{n \rightarrow \infty} z t_{n}=p$ $\notin E(X)$, then $\lim _{n \rightarrow \infty} y t_{n}=p$ for all $y \in Y$. It is said to be $C C$-indivisible by $T$ if in addition whenever there is one $z \in Y$ such that $\left\{z t_{n}\right\}$ has

AMS 1969 subject classifications. Primary 5482; Secondary 5435, 5701.

Key words and phrases. Transformation groups, equicontinuity, connectedness, dimension, manifolds. 
no limit point, then it is true for all $y \in Y$ (the two notions coincide for compact $X$ ).

Theorem 2. (Main Theorem). Suppose $X$ is connected, $N(X)$ $(\neq \varnothing)$ is 0-dimensional and each point in it has a compact orbit closure, and $E(X)$ is indivisible by $T$. Then

(a) $N(X)$ is compact and is the union of one or two indecomposable sets (a set is said to be indecomposable if it is nonempty, closed, invariant and is not the union of two proper subsets with the same properties).

(b) For the two indecomposable sets case, $N(X)$ is a union of two fixed points $\{p, q\}$ and there exists a sequence $\left\{t_{n}\right\}$ in $T$ such that

$$
\lim _{n \rightarrow \infty} x t_{n}=p, \quad \lim _{n \rightarrow \infty} x t_{n}^{-1}=q, \text { for all } x \in E(X) .
$$

(c) For the one indecomposable set case, $N(X)$ is either a fixed point, a Cantor set consisting of a fixed point and dense orbits, a Cantor minimal set or a single orbit which is the union of two points.

For all cases when $X$ is compact, for any $a \in N(X)$ there is a $b \in N(X)$, and a sequence $\left\{t_{n}\right\}$ in $T$ such that

$$
\begin{aligned}
& \lim _{n \rightarrow \infty} x t_{n}=a, \text { for all } x \in X, x \neq b, \\
& \lim _{n \rightarrow \infty} x t_{n}^{-1}=b, \quad \text { for all } x \in X, x \neq a .
\end{aligned}
$$

(d) If $T$ is either connected or abelian, then $N(X)$ is the union of one or two fixed points.

(e) $X$ is separable.

Theorem 3. If $N(X)$ is 0-dimensional, then $E(X)$ is $C C$-indivisible by $T$ if it has a dense subset $D$ which satisfies one of the following conditions:

(I) $D$ is a semicontinuum (i.e. every two points can be joined by a continuum).

(II) $D$ is locally compact, connected, and invariant (this includes the case when $N(X)$ is closed and $E(X)$ is connected; the condition "invariant" can be omitted if $T$ is connected).

(III) $D$ is connected and locally connected.

(IV) $D$ is connected and $N(X)$ is a discrete space.

(V) $X$ is uniformly locally compact (i.e. there exists $\epsilon>0$ such that every closed $\epsilon$-ball at every point is compact) and $D$ is the union of a family $\left\{M_{\lambda}\right\}$ of connected sets, which are totally ordered by inclusions and which satisfy $d\left(M_{\lambda}, N(X)\right)>0$ for all $\lambda$, where $d$ is the metric on $X$. 
THEOREM 4. If $X$ is a connected metrizable manifold of dimension $\geqq 2$, the manifold may or may not be separable and may or may not have boundary. Let, with respect to a certain metric on $X$, the set $N(X)$ be nonempty 0-dimensional and its every point have a compact orbit closure. Then $N(X)$ is compact and all other conclusions of Theorem 2 hold.

Definition. The transformation group $(X, T, \pi)$ is said to be (strictly) almost equicontinuous if $X$ is connected separable, $N(X)$ is (nonempty and) 0 -dimensional compact and $E(X)$ is $C C$-indivisible by $T$ (for having the last condition it is sufficient that $E(X)$ is connected).

THEOREM 5. If $(X, T, \pi)$ is strictly almost equicontinuous but $X$ is not compact, then $N(X)$ is a fixed point.

We define the spread function $\left(f_{T}: X \rightarrow[0, \infty]\right)$ by

$$
f_{T}(x)=\inf \left\{\sup \left\{\operatorname{diameter}\left[S_{\epsilon}(x) t\right] \mid t \in T\right\} \mid \epsilon>0\right\},
$$

where $S_{\epsilon}(x)$ denotes the $\epsilon$-open ball at $x$. Then $x \in N(X)$ iff $f_{T}(x) \neq 0$.

Theorem 6. Let $X$ be a compact connected metric space, $N(X)$ a closed nonempty subset and $E(X)$ connected. Suppose either

(1) there exists some $x \in E(X)$ which is not an almost periodic point, or

(2) $\max \left\{f_{T}(x) \mid x \in X\right\}>\sup \{$ diameter $C \mid C$ components of $N(X)\}$. Then

(a) $N(X)$ has at most two invariant components, and if $T$ is abelian there is at least one invariant component.

(b) If $T$ is connected, then $N(X)$ has exactly one or two components.

(c) The set of periodic points of $(X, T, \pi)$ is contained in two, possibly one, of the components of $N(X)$.

(d) If $N(X)$ has only finite number of components, then the number is at most two.

Suppose $T$ is abelian and $(X, T, \pi)$ satisfies

(3) $\inf \left\{f_{T}(x) \mid x \in N(X)\right\}>\sup \{$ diameter $C \mid C$ components of $N(X)\}$.

Then $N(X)$ has exactly one or two components and they are invariant.

Theorem 7. Let $(X, T, \pi)$ have the same hypotheses as the main theorem. If $N(X)$ is not the union of two points, then $P(X)=X \times X$, where $P(X)$ is the proximal relation in $X$ (i.e. the set of $(x, y) \in X \times X$ s.t. $\lim \inf \{d(x t, y t) \mid t \in T\}=0)$. Therefore, the Cantor minimal set in (c) of Theorem 2 is totally minimal (minimal under every normal subgroup $S$ of $T$ such that there is a compact subset $K, S K=T)$. If $N(X)$ is 
the union of two points $p$ and $q$, then $P(X)=X \times X-\{(p, q),(q, p)\}$.

Theorem 8. Suppose $(X, T, \pi)$ is an almost equicontinuous transformation group and $T$ is a generative group, then there exist $a \in T$ such that $N(X, d, T)=N(X, d,[a])$, where $[a]=\left\{a^{n} \mid n=0,+1, \cdots\right\}$.

THEOREM 9. Let $X$ be a connected metrizable $n$-manifold $(n \geqq 2)$, which is not assumed to be second countable to begin with. If $X$ admits a transformation group $(X, T)$, which has the property that with respect to a certain metric of $X$ the set $N(X)$ is 0-dimensional nonempty and the orbit closure of its every point is compact, then the following statements hold:

(I) If $X$ is compact without boundary, then $X$ is topologically an $n$ sphere.

(II) If $X$ is noncompact without boundary, then $X$ is topologically the Euclidean $n$-space.

(III) If $X$ is compact with boundary, then $X$ is topologically the manifold obtained from deleting open one disk from the $n$-sphere.

(IV) If $X$ is noncompact with boundary, then $X$ is topologically the manifold obtained from deleting open one disk from the Euclidean nspace.

Part (I) of Theorem 9, when $(X, T, \pi)$ is a discrete flow, is proved by K. Kinoshita [9]. Our proof made use of two theorems of Morton Brown [2], [1]:

(a) monotone union of a sequence of open $n$-cells is an open $n$-cell,

(b) a compact manifold which is the union of two open $n$-cells is the $n$-sphere.

THEOREM 10. Let $M$ be a second countable connected 2-manifold without boundary. Conditions such as compactness or orientability are not assumed. Let there be a continuous flow on $M$ which is equicontinuous at every point with respect to one of the metrics on $M$, and let $F(M)$ denote its set of fixed points. Then one and only one of the following three situations must occur:

(1) $F(M)=M$.

(2) $F(M)=\varnothing$.

(3) $M$ is decomposed into a union of periodic orbits and one, but at most two, of the orbits are fixed points.

Moreover, if $M$ has exactly two fixed points then it is topologically the 2-sphere; if $M$ has exactly one fixed point then it is topologically the Euclidean plane or the projective plane.

The details of our work appear somewhere else. 


\section{REFERENCES}

1. Morton Brown, A proof of the generalized Schoenflies theorem, Bull. Amer. Math. Soc. 66 (1960), 74-76. MR 22 \#8470b.

2. - The monotone union of open n-cells is an open n-cell, Proc. Amer. Math. Soc. 12 (1961), 812-814. MR 23 \#A4129.

3. W. H. Gottschalk and G. A. Hedlund, Topological dynamics, Amer. Math. Soc. Colloq. Publ., vol. 36, Amer. Math. Soc., Providence, R. I., 1955. MR 17,650.

4. W. J. Gray and F. A. Roberson, On the near equicontinuity of transformations groups, Proc. Amer. Math. Soc. 23 (1969), 59-63. MR 39 \#7583.

5. T. Homma and S. Kinoshita, On homeomorphisms which are regular except for a finite number of points, Osaka Math. J. 7 (1955), 29-38. MR 16,1140.

6. S. K. Kaul, On almost regular homeomorphisms, Canad. J. Math. 20 (1968), 1-6. MR 36 \#5908.

7. B. V. Kerékjárto, Topologische Charakterisierung der linearen Abdildungen, Acta Sci. Math. (Szeged) 6 (1934), 235-262.

8. S. Kinoshita, On quasi translations in 3-space, Topology of 3-Manifolds and Related Topics (Proc. Univ. of Georgia Inst., 1961), Prentice-Hall, Englewood Cliffs, N. J., 1962, pp. 223-226. MR 25 \#3116.

9. - On quasi-translations in 3-space, Fund. Math. 56 (1964), 69-79. MR $30 \# 1502$.

10. D. Montgomery and L. Zippin, Topological transformation groups, Interscience Tracts in Pure and Appl. Math., no. 1, Interscience, New York, 1955. MR 17,383.

University of Missouri, Columbia, Missouri 65201 